\title{
Demonstration of an Area-Enclosing Guided-Atom Interferometer for Rotation Sensing
}

\section{Citation}

Wu, Saijun, Edward Su, and Mara Prentiss. 2007. "Demonstration of an Area-Enclosing Guided-Atom Interferometer for Rotation Sensing." Physical Review Letters 99 (17). https:// doi.org/10.1103/physrevlett.99.173201.

\section{Permanent link}

http://nrs.harvard.edu/urn-3:HUL.InstRepos:41467465

\section{Terms of Use}

This article was downloaded from Harvard University's DASH repository, and is made available under the terms and conditions applicable to Other Posted Material, as set forth at http:// nrs.harvard.edu/urn-3:HUL.InstRepos:dash.current.terms-of-use\#LAA

\section{Share Your Story}

The Harvard community has made this article openly available.

Please share how this access benefits you. Submit a story.

Accessibility 


\title{
Demonstration of a moving guide based atom interferometer for rotation sensing
}

\author{
Saijun $\mathrm{Wu}$, Edward $\mathrm{Su}$, Mara Prentiss \\ Department of Physics, Harvard University, Cambridge, MA, 02138 and \\ School of Engineering and Applied Science, Harvard University, Cambridge, MA, 02138
}

(Dated: October 18, 2018)

\begin{abstract}
We demonstrate area-enclosing atom interferometry based on a moving guide. Light pulses along the free propagation direction of a magnetic guide are applied to split and recombine the confined atomic matter-wave, while the atoms are translated back and forth along a second direction in $50 \mathrm{~ms}$. The interferometer is estimated to resolve ten times the earth rotation rate per interferometry cycle. We demonstrate a "folded figure 8" interfering configuration for creating a compact, large-area atom gyroscope with multiple-turn interfering paths.
\end{abstract}

PACS numbers: $39.20+q$ 03.75.dg

In a Sagnac interferometry based gyroscope, a rotation with an angular velocity $\Omega$ induces a shift of the interference fringe by the Sagnac phase $\varphi_{\text {Sagnac }}=\frac{2 m \Omega A}{\hbar}$, with $m$ the total mass of the interfering particle, $\hbar$ the reduced Planck constant, and $A$ the area enclosed by the interfering paths projected along the rotation axis. Due to the large total mass, the Sagnac phase for atoms with the same area $A$ is typically $10^{10}$ times larger than it is for visible photons, which makes the atomic matter-wave gyroscope a promising candidate [1, 2] to replace laser gyroscopes [3] to deliver unprecedented rotational sensitivities, with applications expected in long-distance inertial navigation, in geophysics research, and potentially in testing fundamental theories such as to measure geodesic and frame-dragging effects [1].

Existing atom gyroscopes use diffractive optics to manipulate atomic beams. In a typical 3-grating setup [2], the area enclosed by the interfering paths can be expressed as $A=\frac{1}{2} v_{s} \times v_{\text {beam }} T^{2}$, with $v_{s}$ the recoil velocity associated with the grating diffraction, $v_{\text {beam }}$ the atomic beam velocity, and $T$ the time during which the atoms are successively interrogated by the three gratings. For example, the state-of-the-art atom gyroscope developed in the Kasevich group [4] uses cesium atomic beams with an enclosed-area of $A \sim 22 \mathrm{~mm}^{2}$, based on an apparatus that is $L=v_{\text {beam }} T \sim 2 \mathrm{~m}$ long. Further increasing the area of atomic gyroscopes would improve their rotational sensitivity. However, atom gyroscopes need to be as compact as possible to suppress phase shifts due to stray fields as well as those due to mechanical vibrations, and a longer apparatus would limit the applications of the gyroscope. Recent interferometry techniques have been developed with slow atoms in parabolic trajectories [5]. However, a significant improvement of the sensing area in fountain geometry requires a tall atomic fountain and thus a large apparatus.

An obvious solution to fulfill the contradictory requirements of being compact and having a large sensing area is to let the two interfering paths circulate around a small

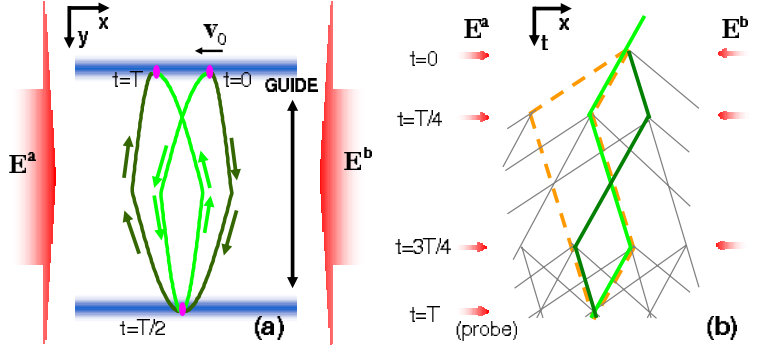

FIG. 1: (Color online) (a) Schematic of a moving guide interferometer. The two blue bars represent the position of the moving guide at times $t=0, T$ (top) and $t=T / 2$ (bottom), with the center-of-mass position of a guided atomic wavepacket at the three times marked. The optical fields $\mathbf{E}^{a}$ and $\mathbf{E}^{b}$ are pulsed (see b). The atom follows the thick solid curve to interfere at time $t=T$. (b) The $\mathrm{x}$-t recoil diagram for the 4-pulse scheme. At time $t=T$ the atomic fringe is probed via a Bragg-scattering from $\mathbf{E}^{a}$ to $\mathbf{E}^{b}$. The "figure 8" interfering loop marked with thick solid lines corresponds to that in (a). Another loop marked with dashed lines is referred to as a "trapezoid" loop.

physical area multiple times. To suppress any phase shift due to static perturbations, the paths may be chosen to be reciprocal, with one path following the time-reversal path of the other. A multiple-turn reciprocal interference configuration may be achievable using atomic guiding potentials. Recent research efforts have realized atomic wave-guide potentials in closed loops [ $[\underline{6}$. However, interferometry with these devices has not been demonstrated. One of the technical challenges of this approach stems from the dispersive coupling of motion between the confining direction and the guide direction in curved atomic wave-guides [7], usually large even in the adiabatic limit. Recently the interference of a propagating Bose-Einstein condensate was demonstrated to enclose an area following a "Y" splitting scheme [8]; however, it is unclear how to use the scheme to enclose a large area in multiple-turn reciprocal geometry.

Instead of transporting atoms with curved waveguides, we demonstrate an area-enclosing guided atom 
interferometer in a "folded figure 8" configuration based on a straight guide [9] that moves. In particular, a two-dimensional interfering path of guided atoms is created by a pulsed optical standing wave field along $\mathbf{e}_{\mathbf{x}}$ combined with a guiding potential oscillating along e $\mathbf{y}$ (Fig. 17). A 4-pulse de-Broglie wave interferometry scheme (Fig. 1b) [10, 11] is applied to create an effectively-reciprocal interfering loop that encloses an area up to $0.2 \mathrm{~mm}^{2}$ with stable phase readouts. We argue that the scheme demonstrated in this work can be extended to a practical large-area guided atom gyroscope with multiple-turn reciprocal paths.

We consider the schematic setup in Fig. 1. Atoms are confined in a guiding potential oriented to $\mathbf{e}_{\mathbf{x}}$, the direction along which the guiding potential is invariant. An optical standing wave field composed of traveling light $\mathbf{E}^{a}$ and $\mathbf{E}^{b}$ is aligned parallel to $\mathbf{e}_{\mathbf{x}}[9]$. Standing wave pulses split and recombine the atom wavepackets along $\mathbf{e}_{\mathbf{x}}$ by transferring photon recoil momentum to atoms. When atoms are transported along the direction $\mathbf{e}_{\mathbf{y}}$, the interfering paths enclose the area in the $\mathbf{x}-\mathbf{y}$ plane. In particular, as represented by the recoil diagrams shown by the thick solid lines in Fig. 1 $\mathrm{k}$, b, a nearly reciprocal interfering loop can be created by setting the guide velocity $v_{y} \propto \sin \left(\frac{2 \pi t}{T}\right)$ while the atom wavepackets are split at time $t=0$ and redirected at time $t=T / 4$, $3 T / 4$. Pairs of wavepackets meet at time $t=T$, creating a "folded figure 8" loop in the x-y diagram and a "figure 8" loop in the x-t diagram. The area enclosed by the loop is $A=\frac{L}{\pi} \delta v_{x} T$, where $\delta v_{x}$ is the wavepacket splitting velocity due to photon recoils and $L$ is the maximum translation distance along $\mathbf{e}_{\mathbf{y}}$. Since the atoms almost return to the initial position at time $T$, the sequence may be repeated $N$ times; each time the guide passes the half distance $L$ the two interfering paths are deflected by an additional standing wave pulse, resulting in multiple turn interfering paths. Precise reciprocity of the interfering paths can be achieved with atoms starting with zero velocity. With non-zero initial velocity $v_{0}$ (Fig. 17), effective reciprocity is retained that suppresses the interferometry phase shifts due to potential variations at the length scale $l$ with $v_{0} T<<l$.

In this demonstration, a 4-pulse de-Broglie wave interferometry scheme (Fig. 1b) [10, 12] is applied to address most of the atoms in our $25 \mu K$ atomic sample. We use short standing wave pulses (300 ns width) as thin phase gratings, that diffract atoms to multiple diffraction orders weighted by the amplitude $i^{n} J_{n}(\theta)$. Here $\theta$ is the standing wave pulse area and $J_{n}$ is the $n^{\text {th }}$ order Bessel function [10]. Pairs of paths interfere and create an atomic density grating at time $T$, which is probed by monitoring the Bragg scattering of a probe light from $\mathbf{E}^{a}$ into $\mathbf{E}^{b}$ mode [11]. The two requirements for any two interfering paths to contribute to the Bragg backscattering of a probe light (grating echo) are: 1) they meet at time $T$ to form a loop, and 2) at time $T$ their rela-

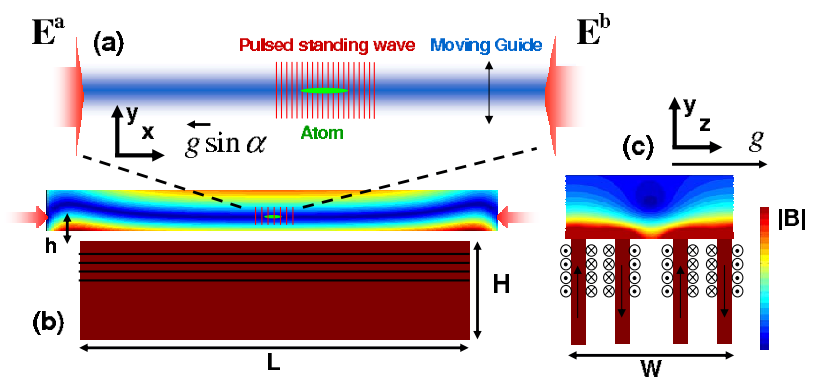

FIG. 2: Schematic of the moving guide interferometry setup in this work. (a) is a zoom-in of (b) at the standing wave zone. (b) and (c) show the arrangement of the permalloy foils and the current-carrying wires. A density plot of the simulated magnetic guiding potential is included in (b) and (c).

tive velocities should be twice the atomic recoil velocity $2 v_{r} \approx 11.8 \mathrm{~mm} / \mathrm{sec}$. We classify these loops according to the relative displacements between each pair of paths. In addition to the "figure 8" loop in Fig. 10 that corresponds to the "folded figure 8" loop in Fig. 19, other interfering loops also contribute, such as the "trapezoid" loop in Fig. 1 b. The final interferences are composed of the contributions from all these loops. It can be shown that the contributions from the "figure 8 " and the "trapezoid" loops are weighted by $J_{2}^{2}(2 \theta)$ and $J_{1}^{2}(2 \theta)$ respectively [11], while other loops contribute negligibly for standing wave pulse area $\theta<2$. The reciprocity of the figure 8 loop ensures a zero differential phase shift between the two paths in the presence of a linear potential along $\mathbf{e}_{\mathbf{x}}$, and suppresses the dephasing effects due to nonlinear potential variations. This is in contrast to the trapezoid loop that acquires a phase shift of $\varphi(T)=\frac{3}{8} k a T^{2}$ due to an acceleration force $m a \mathbf{e}_{\mathbf{x}}$ ( $k$ the wave-vector of the light field) and dephases quickly in a nonlinear potential. The reciprocity of the "figure 8" loop is confirmed experimentally by analyzing the readouts due to the "trapezoid" loop and the "figure 8" loop.

In what follows, we briefly summarize our experimental apparatus, detailed in [11]. The layout of the experimental setup is described in Fig. 2. Four $200 \mathrm{~mm}$ $\times 100 \mathrm{~mm} \times 1.5 \mathrm{~mm}$ permalloy foils, separated by $6.35,12.7$ and $6.35 \mathrm{~mm}$, are poled in alternating directions (Fig. 2 $\mathrm{b}$, c) to generate a quadruple field as the guiding potential. Close to the center of the foils, the quadruple field can be approximately described by $\mathbf{B}=B_{1}\left(z \mathbf{e}_{y}+y \mathbf{e}_{z}\right)+B_{0} \mathbf{e}_{\mathbf{x}}$. A wave-guide operation distance of $h \sim 7 \mathrm{~mm}<<L=200 \mathrm{~mm}$ is chosen to minimize the edge effects. With the wave-guide operating at a field gradient of $B_{1}=70 \mathrm{G} / \mathrm{cm}, B_{0}$ varies about $10 \mathrm{mG}$ over a centimeter along $\mathbf{e}_{\mathbf{x}}$, likely due to the foil-surface inhomogeneities. Approximately $10^{7}$ laser-cooled ${ }^{87} \mathrm{Rb}$ atoms in their ground state $F=1$ hyperfine level are loaded into this magnetic guide resulting in a cylindrically-shaped atom sample $1 \mathrm{~cm}$ long and $170 \mu \mathrm{m}$ wide. The standing 


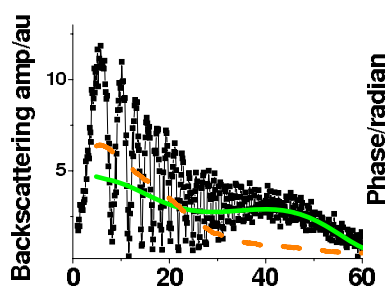

(a) $\mathrm{T} / \mathrm{ms}$

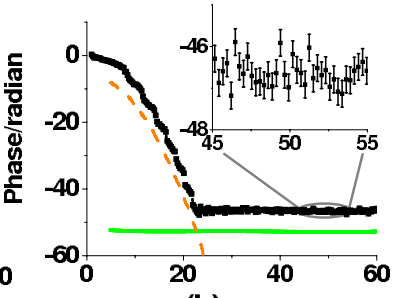

(b) $T / m s$
FIG. 3: (Color online) 4-pulse interferometry readout in a stationary guide. The 4-pulse data (black) is decomposed into the solid and the dash curves, that corresponds to the contributions from the "figure 8" and "trapezoid" loops in Fig. 1 respectively. (a) Interferometry amplitude (b) Interferometry phase with an inset plot around $50 \mathrm{~ms}$. The phases in (b) have been unwrapped. The error bars give the phase noise due to mirror vibrations.

wave is formed by two counter-propagating laser beams $\mathbf{E}^{a}$ and $\mathbf{E}^{b}(120 \mathrm{MHz}$ to the blue side of the $\mathrm{F}=1$ $\mathrm{F}^{\prime}=2 \mathrm{D} 2$ transition) with diameters of $1.6 \mathrm{~mm}$. A heterodyned detection of the Bragg scattering is applied to retrieve both the amplitude and the phase of the atomic fringe [10]. Precise alignment of the standing wave $\mathrm{k}$ vector parallel to the free-propagation direction of the guide is required to decouple the wave-guide confinement from the interferometry phase readouts [11]. To ensure parallelism, the relative angle between the standing wave and the wave-guide is minimized with two rotation stages (with the rotation axis along $\mathbf{e}_{\mathbf{y}}$ and $\mathbf{e}_{\mathbf{z}}$ in Fig. 2) at a precision better than $0.2 \mathrm{mrad}$ by minimizing the confinement-induced interferometry dephasing [13].

To illustrate the interference and decay of different interfering loops in Fig. 1 b, we first discuss interferometry output with atoms in the stationary guide that are shown in Fig. 3. Given the 300 ns standing wave pulse duration, we set the pulse area to be $\theta=1.3$ for the second and the third pulse by adjusting the intensity of the pulses. In repeated experiments, the amplitude and phase of the Bragg scattering signals were recorded with different total interrogation time $T$, which is incremented from $T=0.9945 \mathrm{~ms}$ to $T=60.1314 \mathrm{~ms}$ in steps of $132.6 \mu \mathrm{s}$. The oscillatory amplitude/phase readout in Fig. 3 is due to the beat between the "figure 8" loop (thick solid line) and the "trapezoid" loop ( thick dashed line) in Fig. 10. With a numerical routine to separate the fast oscillating parts from the slowly varying parts, the oscillatory interferometry amplitude and phases are decomposed to the solid and dashed curves in Fig. 3, which represent the contributions from the "figure 8" and the "trapezoid" loops in Fig. 1b respectively. The acceleration force is due to a small gravity component along the direction $\mathbf{e}_{\mathbf{x}}$ with $a=g \sin \alpha$ (In Fig. 2 the gravity is along $\mathbf{e}_{\mathbf{z}}+\alpha \mathbf{e}_{\mathbf{x}}$ and $\alpha \sim 3 \mathrm{mrad}$ ). We extract the acceleration constant $a=31.0(5) \mathrm{mm} / \mathrm{sec}^{2}$ from the dashed curve in Fig. 3 $\mathrm{p}$, which is found to agree with those from inde-

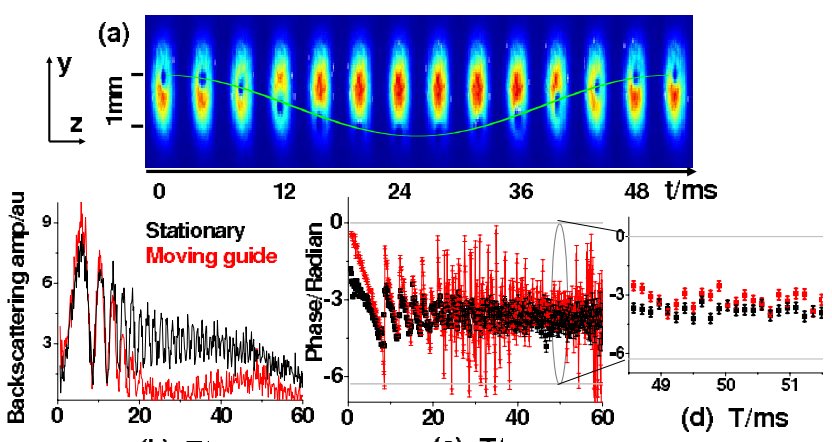

(b) $\mathrm{T} / \mathrm{ms}$

(c) $\mathrm{T} / \mathrm{ms}$

FIG. 4: Top: Absorption images of the guided atomic sample following the moving guide in $50 \mathrm{~ms}$. The graphs are expanded along the $\mathbf{e}_{\mathbf{y}}$ direction for clarity. A "cosine" curve is added as a guide for the readers' eyes. Bottom: Interferometer signals for the case of stationary (black) and moving (red) guides. In (c), (d) the error bars indicate the standard phase deviation due to mirror vibrations. Notice the scale difference between Fig. 3b and (c) here.

pendent measurements using 3-pulse interferometers [10]. The solid curve in Fig. 3 a has a smaller initial amplitude but a slower decay, so that the two curves cross at $T \sim 22 \mathrm{~ms}$. Correspondingly, the phase readout in Fig. 3 approximately follows the parabolic curve for $T<22 \mathrm{~ms}$ but is locked to a constant value for $T>22 \mathrm{~ms}$. The slow dephasing of the "figure 8" loop is attributed to the approximate reciprocity between the two interfering paths (Fig. 10). The results in Fig. 3] demonstrate that in the presence of multi-loop interference in this experiment, an independent observation of the "figure 8" loop is nevertheless possible for $T \sim 50 \mathrm{~ms}$ due to its relatively long coherence time.

We now discuss the moving guide interferometer. To translate the atomic sample along $\mathbf{e}_{\mathbf{y}}$, we pulse a $50 \mathrm{~ms}-$ period sinusoidal current through the coils that magnetize the inner two foils of the 4 -foil (Fig. 26). The resulting motion of the guided atomic sample in the $\mathbf{y}-\mathbf{z}$ plane is monitored with the absorption images probed by $\mathbf{E}^{\mathbf{b}}$ (Fig. 2a, b). For the absorption image, the frequency of $E^{b}$ is tuned to resonance before the laser is coupled into a fiber. The fiber coupling ensures that the spatial mode of $E^{b}$ is consistent for both the absorption image and the standing wave formation. A sequence of absorption images taken in repeated experiments is presented in Fig. 4 a. The motion of atoms follows a "cosine" trace fairly well with an amplitude of 0.97(5) $\mathrm{mm}$.

To realize area-enclosing interferometry, we repeat the 4-pulse interferometry measurements with the same current settings that induced the atomic motion shown in Fig. 4a. Typical interferometry amplitude and phase readouts are plotted in Fig. 4 in red, together with comparison data from another stationary guide experiment plotted in black. Here a standing wave pulse area of 
$\theta=1.5$ is chosen to maximize the interference due to the "figure 8" loop. A consequence is the more constant phase readout for the black plots in Fig. 44, compared with those shown in Fig. 3b. In contrast to the stationary guide case, the amplitude of the moving guide interferometer signal (red plots) almost vanishes in the time window of $T$ from $25 \mathrm{~ms}$ to $35 \mathrm{~ms}$ because the atoms are at the very outer edge of the optical fields when the probing pulse is fired, resulting in vanishing backscattering signals and thus random phase readouts (Fig. 4 4 ). For $T \sim 50 \mathrm{~ms}$, the atomic sample returns back to within the $\sim 1.6 \mathrm{~mm}$ diameter of the optical fields. The backscattered signal revives and the phase readout matches those

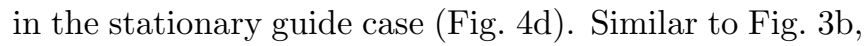
the constant and repeatable phase readouts in Fig. $4 \mathrm{~d}$ are due to the approximate reciprocity of the "figure 8" loop, which in the spatial-domain becomes the "folded figure 8" loop in Fig. 1 1 . For $T=50 \mathrm{~ms}$, the interferometer encloses an area of $0.18 \mathrm{~mm}^{2}$ 14. A rotation rate of $1 \mathrm{mrad} / \mathrm{sec}$ should induce a Sagnac phase of $\pi$ that shifts the red plot in Fig. 4 d.

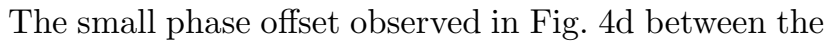
readouts from a stationary guide and the moving guide is repeatable before any realignments. The offset is due to the guiding potential variation along $\mathbf{e}_{\mathbf{x}}$. In this experiment, the moving guide potential does not precisely follow a sinusoidal trace, and collective atomic oscillations in the moving guide are expected since the waveguide confinement is relatively loose. The non-reciprocity leads to imperfect cancelation of the phase shifts before and after $t=T / 2$ (Fig. (1) that results in the phase offset in Fig. 4h. The imperfection is not precisely repeatable in each experimental trial, resulting in a standard deviation of the phase readout $\sim 0.4 \mathrm{rad}[15]$. A smoother and more precisely controlled guiding potential would help to suppress the phase error as well as the dephasing effects that have limited the interrogation time of $50 \mathrm{~ms}$ in this experiment. By reducing the wavepacket separations using a different interferometry scheme, we have achieved up to $1 \mathrm{sec}$ coherence time with guided atoms [16].

The $\sim 0.2 \mathrm{~mm}^{2}$ enclosed area is much smaller than in [4]. However, orders of magnitude improvements in the sensing area are expected with larger guide translation distances, a more efficient beamsplitting scheme, and longer interrogation time [11]. To increase the waveguide translation distance, a multi-wire $1 \mathrm{D}$ conveyer belt on an atom chip [9] may be constructed. The localized atomic sample in the wave-guide should facilitate a high-efficiency multiple-recoil beamsplitting [17] where the light intensity control is important. We consider a ${ }^{87} \mathrm{Rb}$ atomic sample at sub-recoil temperatures so that multi-photon beam splitting techniques can be applied giving efficient $\pm 6 \hbar k$ momentum splittings [17]. If the wave-guide were oscillated $5 \mathrm{~cm}$ back and forth 5 times accompanied by 10 appropriately timed Bragg pulses, the interferometer on a centimeter-scale device would enclose an area greater than $\sim 1000 \mathrm{~mm}^{2}$ in a second. If we consider a shot-noise limited phase resolution, the resulting on-chip atom gyroscope would have a rotational sensitivity of $1 \times 10^{-9} \mathrm{rad} / \mathrm{sec} \sqrt{\mathrm{Hz}}$ comparable to [4], even with only $10^{4}$ atoms per experimental cycle. Parallel operation of multiple guided atom gyroscopes may further boost the measurement bandwidth and sensitivity.

In conclusion, we have demonstrated a moving-guide based atom interferometer that encloses an area of $0.2 \mathrm{~mm}^{2}$ and has stable phase readout for rotation sensing. We have demonstrated a "folded figure 8" interferometry configuration whose reciprocity partly suppresses matterwave dephasing due to guiding potential variations. The "folded figure 8" configuration should be sensitive to rotation but extremely insensitive to linear acceleration. The scheme may be extended to enable sensitive rotation measurement with a multiple-turn atomic matterwave gyroscope in a compact device.

This work also demonstrates coherent transportation of matter-waves using magnetic-dipole forces. The moving guide may allow a light pulse atom interferometer to interact with a distant object where the optical path is restricted, such as to sense the light shift of a micro-cavity or a surface potential.

This work is supported by MURI and DARPA from DOD, NSF, ONR and U.S. Department of the Army, Agreement Number W911NF-04-1-0032, and by the Charles Stark Draper Laboratory. We thank the referees for their suggestions on the earlier version of this article.

[1] J. F. Clauser, Physica B, 151, 262 (1988).

[2] F. Riehle et al., Phys. Rev. Lett. 67, 177 (1991). A. Lenef et al., Phys. Rev. Lett. 78, 760 (1997). T. L. Gustavson et al., Phys. Rev. Lett. 78, 2046 (1997).

[3] G. E. Stedman, Rep. Prog. Phys. 60, 615 (1997).

[4] T. L. Gustavson et al., Classical Quantum Gravity 17, 2385 (2000). D. S. Durfee et al., Phys. Rev. Lett. 97, 240801 (2006).

[5] B. Canuel et al., Phys. Rev. Lett. 97, 10402 (2006).

[6] J. Sauer et al., Phys. Rev. Lett. 87, 270401 (2001). S. Wu et al., Phys. Rev. A 70, 013409 (2004). S. Gupta et al., Phys. Rev. Lett. 95, 143201 (2005).

[7] K. W. Murch et al., Phys. Rev. Lett. 96, 013202 (2006).

[8] G.-B. Jo et al., Phys. Rev. Lett. 98, 030407 (2007).

[9] Y.-J. Wang et al., Phys. Rev. Lett. 94, 090405 (2005).

[10] S. B. Cahn et al., Phys. Rev. Lett. 79, 784 (1997).

[11] S. Wu, Ph. D. Thesis, Harvard Univ. (2007).

[12] T. W. Mossberg et al., Phys. Rev. Lett. 43, 851 (1979).

[13] S. Wu et al., Eur. Phys. J. D 35, 111 (2005).

[14] Area up to $0.2 \mathrm{~mm}^{2}$ was enclosed with $T=54 \mathrm{~ms}$.

[15] The phase noise due to imperfect suppression of the "trapezoid" loop is $\sim 0.2 \mathrm{rad}$ here. A method to completely supress the loop was suggested by B. Dubetsky et al. in Phys. Rev. A 74, 023615 (2006).

[16] E. J. Su et al., physics/0701018.

[17] S. Wu et al., Phys. Rev. A 71, 43602 (2005). 